\title{
Dispersion Anisotropy of Plasmon-Exciton-Polaritons in Lattices of Metallic Nanoparticles
}

\author{
Mohammad Ramezani, ${ }^{*}{ }^{\dagger}$ Alexei Halpin, ${ }^{\dagger}$ Johannes Feist, ${ }^{\ddagger}{ }^{\circledR 0}$ Niels Van Hoof, ${ }^{\dagger}$ \\ Antonio I. Fernández-Domínguez, ${ }^{\ddagger}$ Francisco J. Garcia-Vidal, ${ }^{\ddagger}, \mathbb{I}$ and Jaime Gómez Rivas ${ }^{\dagger, \S}$ \\ ${ }^{\dagger}$ Dutch Institute for Fundamental Energy Research, DIFFER, P.O. Box 6336, 5600 HH Eindhoven, The Netherlands \\ ${ }^{\ddagger}$ Departmento de Física Teórica de la Materia Condensada and Condensed Matter Physics Center (IFIMAC), Universidad \\ Autónoma de Madrid, E-28049 Madrid, Spain \\ II Donostia International Physics Center (DIPC), E-20018 Donostia/San Sebastian, Spain \\ ${ }^{\S}$ Department of Applied Physics and Institute for Photonic Integration, P.O. Box 513, 5600 MB Eindhoven, The Netherlands
}

ABSTRACT: When the electromagnetic modes supported by plasmonic-based cavities interact strongly with molecules located within the cavity, new hybrid states known as plasmon-exciton-polaritons (PEPs) are formed. The properties of PEPs, such as group velocity, effective mass, and lifetime, depend on the dispersive and spectral characteristics of the optical modes underlying the strong coupling. In this work, we focus on lattice modes supported by rectangular arrays of plasmonic nanoparticles known as surface lattice resonances (SLRs). We show that SLRs arising from different in-plane diffraction orders in the lattice can couple with the molecular excitons, leading to PEPs with distinct dispersions and thus different group velocities. These results illustrate the possibility of tailoring the transport of PEPs through the design of lattices of plasmonic particles.

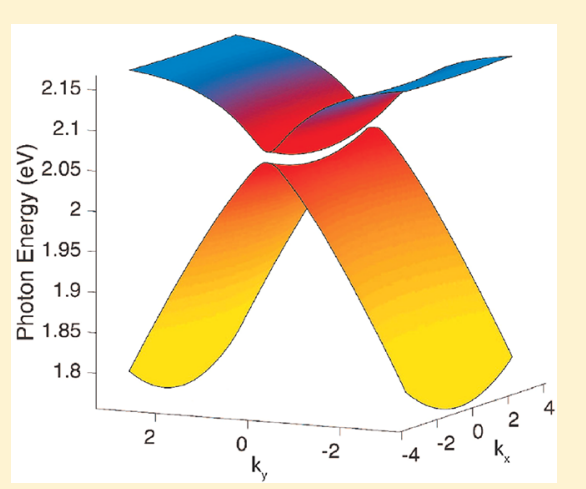

KEYWORDS: strong coupling, exciton-polaritons, plasmonics lattice, organic fluorophores, anisotropic dispersions

$\mathrm{T}$ he realization of strong light-matter coupling at room temperature using organic molecules to exploit properties of exciton-polaritons has been the driving force of many theoretical and experimental studies in recent years. Excitonpolaritons possess intriguing properties that have been exploited for wide-ranging purposes: room-temperature Bose-Einstein condensation and polariton lasing, ${ }^{1-4}$ enhanced exciton transport ${ }^{5,6}$ and conductivity ${ }^{7}$ in organic semiconductors, modified electronic potential energy surfaces in molecular systems, ${ }^{8,9}$ and altered chemical reaction yields ${ }^{10,11}$ constitute a small set of associated research highlights across numerous disciplines.

In the realm of strong light-matter coupling, microcavities have historically been the canonical system for studying exciton-polaritons ${ }^{12}$ by virtue of their intuitive design and robust cavity modes. In recent room-temperature experiments the types of photonic structures employed for strong coupling have multiplied, ranging from single plasmonic nanoparticles ${ }^{13}$ to planar metallic surfaces, ${ }^{14,15}$ plasmonic hole arrays, ${ }^{16}$ and nanoparticle arrays. ${ }^{17,18}$ The hybrid quasi-particles resulting from the strong coupling of molecular excitons with modes in plasmonic resonances, i.e., modes arising from the coherent oscillation of the electrons at the interface between the metal and the surrounding dielectric, are called plasmon-excitonpolaritons (PEPs).

The strength of microcavities lies in the high-quality resonances associated with their well-defined cavity modes.
Plasmonic structures, on the other hand, suffer from strong radiative and nonradiative losses, ${ }^{19}$ resulting in relatively broad line widths. The improvement of the resonance quality factor leads to increased lifetimes, a condition that makes the realization of strongly coupled coherent states feasible. Nevertheless, the strong field enhancement within a small mode volume at the vicinity of these structures has made the realization of strong light-matter coupling possible. ${ }^{20}$

Among the aforementioned plasmonic structures, it has been shown that periodic arrays of plasmonic nanoparticles are suitable platforms for strong coupling experiments. $4,17,18,21-23$ Plasmonic arrays offer the dual advantage of independent control over both the energy-momentum dispersions and the line widths of collective resonances (surface lattice resonances, SLRs) supported by the arrays. ${ }^{24}$ The modification of the dispersion is of central relevance for the design of photonic systems enabling the precise control of the properties of the exciton-polaritons, including group velocity and effective mass. ${ }^{17}$ Furthermore, these systems benefit from an ease in positioning the organic molecules in the vicinity of the plasmonic nanoparticles ${ }^{25}$ and the possibility for integration

Special Issue: Strong Coupling of Molecules to Cavities

Received: July 1, 2017

Published: October 20, 2017 
with planar photonic technologies due to the open nature of the cavity.

In this article, we demonstrate strong coupling of excitons in organic molecules to SLRs in anisotropic arrays of nanoparticles. By exploiting the coupling of excitons to different orders of diffraction, we allow for the excitation of PEPs with different dispersions and group velocities. This leads to an anisotropy in the properties of the generated PEPs which can not take place in a conventional cavity, where cylindrical symmetry limits the dispersion. By combining PEP dispersions with different propagation constants in different directions, it is conceivable to design systems analogous to birefringent crystals for nonlinear polaritonics or provide directivity in the transport and propagation of exciton-polaritons for optoelectronic applications.

\section{SAMPLE PREPARATION}

Arrays of nanoparticles were fabricated by substrate conformal imprint lithography onto glass substrates (Corning Eagle 2000, $n=1.51$ ). This technique, which is based on a polydimethylsiloxane (PDMS) stamp to conformally imprint nanostructures, offers a great potential for fabrication of nanostructures over large areas with high precision and reproducibility. ${ }^{26}$ In this study, we used silver as a metal with low loss in the visible range of the electromagnetic spectrum. A scanning electron micrograph image of the fabricated structure is displayed in the inset of Figure 1. The array of the nanoparticles is a rectangular

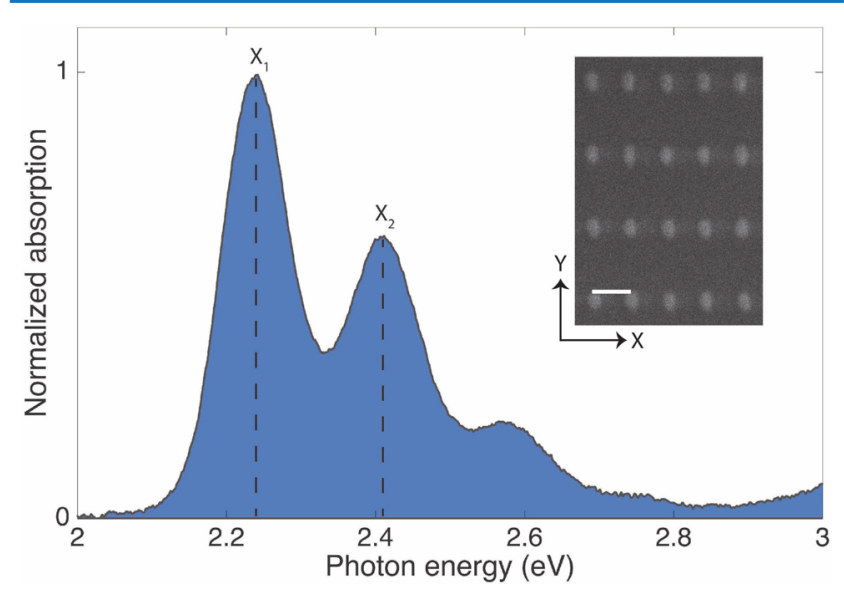

Figure 1. (a) Normalized absorption spectrum of the organic dye. (Inset) SEM image of the array of silver nanoparticles. The scale bar is $200 \mathrm{~nm}$.

lattice with lattice constants $a_{x}=200 \mathrm{~nm}$ and $a_{y}=380 \mathrm{~nm}$ along the short and long axes, respectively. The height of the nanoparticles is $20 \mathrm{~nm}$, and the nominal length and width of the nanoparticles are 70 and $50 \mathrm{~nm}$, respectively.

One complication associated with silver nanoparticles is oxidation under ambient conditions, which can deteriorate their optical and plasmonic responses. To avoid this oxidation, the nanoparticles are encapsulated with an $8 \mathrm{~nm}$ layer of $\mathrm{SiO}_{2}$ and $20 \mathrm{~nm}$ of $\mathrm{Si}_{3} \mathrm{~N}_{4}$ immediately after the evaporation of the silver. These passivation layers lead to stable particles with proper resistance against oxidation.

As an organic dye molecule, we use a rylene-based compound [ $N, N^{\prime}$-bis(2,6-diisopropylphenyl)-1,6,11,16-tetra(2,6 diisopropylphenoxy)-8,18-and-8,19-dibromoquater rylene3,4:13,14-tetracarboximide]. The normalized absorption spec- trum of this compound is shown in Figure 1(a). The ease of processability and high photostability are the main motivations for using this molecule. The main electronic transition of this molecule is located at $E_{X_{1}}=2.237 \mathrm{eV}$, with a line width $\left(\Gamma_{X_{1}}\right)$ of approximately $0.090 \mathrm{eV}$ at room temperature. The second peak at $E_{X_{2}}=2.409 \mathrm{eV}$ corresponds to a vibronic sublevel of the first electronic excited state.

To strongly couple the molecular excitons to the SLRs supported by the array, a layer of poly(methyl methacrylate) (PMMA) containing the molecules with thicknesses of $120 \pm$ $20 \mathrm{~nm}$ was spin-coated on top of the array. Small variations of the thickness within the error range does not influence the optical properties of SLRs. We dissolved the dye molecules and PMMA in chloroform and stirred the solution at $60{ }^{\circ} \mathrm{C}$ for $1 \mathrm{~h}$. Two different samples were prepared with a weight concentration of the organic molecules with respect to PMMA of 35 and 50 wt \%.

\section{SURFACE LATTICE RESONANCES IN ARRAYS OF NANOPARTICLES}

To have a better insight into the electric field distribution of the resonances supported by the metallic nanoparticles, we have performed finite-difference time-domain (FDTD) simulations for the single particle as well as for an array of nanoparticles with the lattice parameters described above. In all the simulations, the structure is illuminated at normal incidence with a plane wave with the polarization vector oriented along the short axis of the nanoparticles. In Figure 2(a), the scattering cross section of the single particle with a resonance at $E_{\mathrm{LSPR}}=$ $2.5 \mathrm{eV}$ is plotted with a red curve. This resonance corresponds to the localized surface plasmon resonance (LSPR) with a dipolar $(\lambda / 2)$ electric field distribution. The total electric field intensities corresponding to the LSPR for the plane across the middle of the particle (top view) and the cross sectional plane (side view) are shown in Figure $2(b, c)$ in a logarithmic scale. The LSPR creates a strong electric field enhancement at the vicinity of the nanoparticles. However, the extension of the field to the surrounding medium is limited to few tens of nanometers. This spatial confinement reduces the efficient coupling of the electromagnetic field to a few of the molecules distributed in the PMMA layer. ${ }^{25}$ Furthermore, the strong ohmic and radiative losses associated with LSPRs lead to the broad line width and short lifetime of these resonances.

The losses associated with LSPRs in individual particles can be significantly reduced by creating a lattice of plasmonic nanoparticles in which the particles interact via coherent scattering by means of the in-plane diffraction orders, known as Rayleigh anomalies (RAs). ${ }^{19,27}$ The RAs lead to the enhanced radiative coupling between LSPRs, resulting in a remarkable modification of the line width and quality factor of the resonance. The resulting modes are the aforementioned SLRs. ${ }^{27,28}$ In this case, the electric field on each particle is the sum of the incident field plus the radiation from all other nanoparticles. The effective polarizability of each nanoparticle in an infinite array is given by ${ }^{28}$

$$
\alpha^{*}=\frac{1}{1 / \alpha-S}
$$

where $\alpha$ is the polarizability of the single particle and $S$ is the retarded dipole sum, which contains the effect of all the other nanoparticles on the polarizability. The extinction cross section 

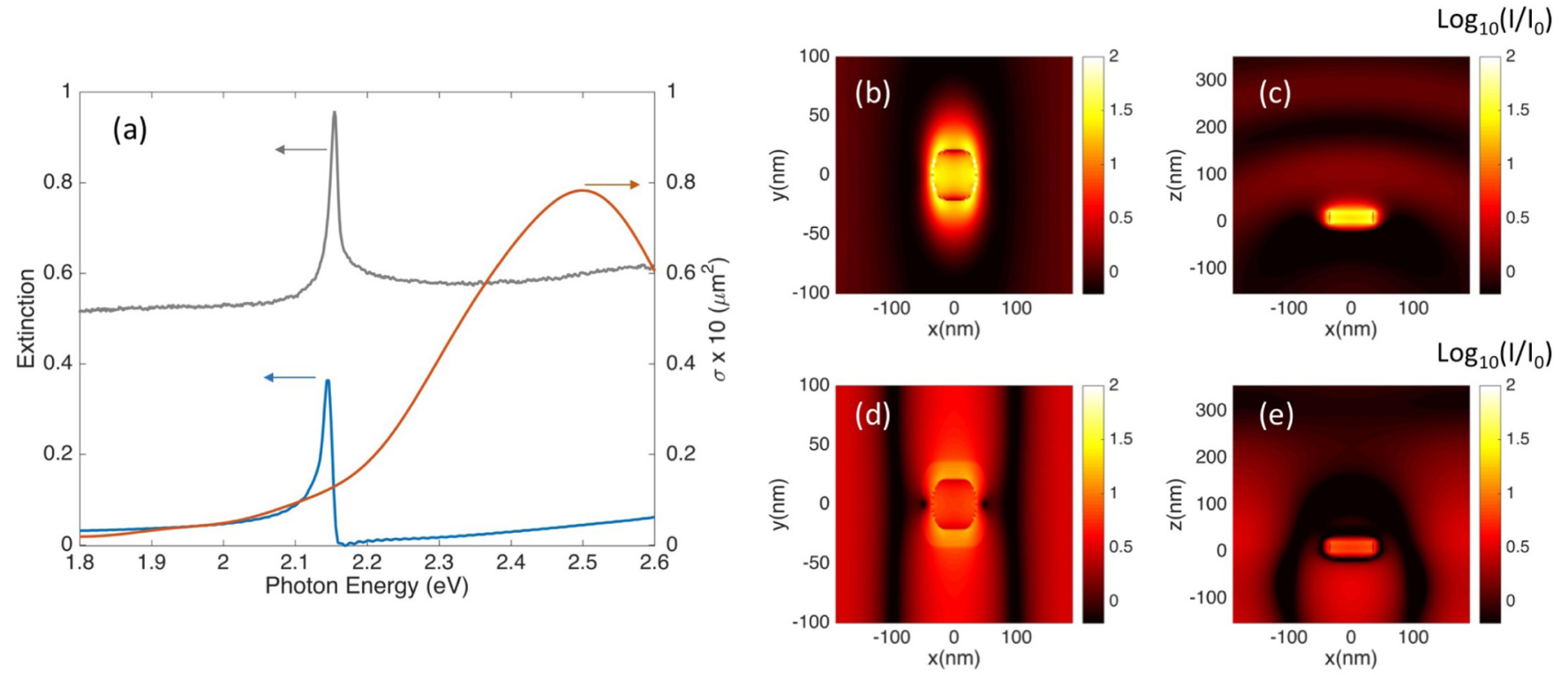

Figure 2. (a) Measurement (gray line) and simulation (blue line) of the extinction for the array of silver nanoparticles. Simulation of scattering cross section of the single particle illuminated by the plane wave (brown line). The extinction measurement is vertically displaced by 0.5 . Electric field intensity of a single particle at $E=2.5 \mathrm{eV}$ at the plane passing through (b) the middle height and (c) the cross section of the particle. Electric field intensity of the particle within the rectangular lattice at $E=2.139 \mathrm{eV}$ for the plane passing (d) through the middle height and (e) the cross section of the particle. Note that in all simulations the incident plane wave is polarized along the short axis of the nanoparticle (along the $y$-direction in panels (b) and (c)).

of the array of coupled particles in terms of effective polarizability can be written as

$$
C_{\mathrm{ext}}=4 \pi k \operatorname{Im}\left(\alpha^{*}\right)
$$

The simulated extinction of the array supporting the enhanced diffractive coupling between LSPRs is shown in Figure 2(a) as a blue curve. The extinction is defined as (1 $\left.T / T_{\text {ref }}\right)$ where $T$ is the zeroth-order transmission, i.e., transmission in the forward direction from the array, and $T_{\text {ref }}$ is the transmission from the sample in the absence of the nanoparticles, i.e., the transmission of the substrate. The appearance of a narrow peak in the extinction of the periodic array of nanoparticles can be explained in terms of interplay between $S$ and $1 / \alpha$. The measured extinction at normal incidence is displayed in Figure $2(\mathrm{a})$ as a gray line. The measurement is shifted vertically for clarity, and it shows a good agreement with the simulations. The small discrepancies between the simulated and measured extinction spectra can be explained by the Gaussian beam illumination and finite sample size in the experiment, compared to the plane wave illumination and infinite sample size for the simulations. Moreover, small size variations between the simulated and measured nanoparticles could also induce some discrepancies.

A significant reduction of the line width (enhanced quality factor) is observed for the array of nanoparticles. Moreover, due to the photonic nature of the diffraction order giving rise to the SLRs, these modes exhibit a more spatially extended electric field profile than LSPRs. Given the fact that the lattice is formed by plasmonic nanoparticles with large polarizability, the local electric field intensity of the modes is enhanced due to LSPRs. This effect can be revealed through the comparison between the spatial distribution of electric field intensity for a single nanoparticle (Figure $2(b, c)$ ) and the nanoparticle in the lattice (Figure 2(d,e)). In Figure 2(d,e), we can see the simultaneous delocalization and enhancement of the electric field intensity in the lattice. The spatial modification of the electromagnetic field and its further extension into the regions far from the particle lead to an increase in the number of molecules that can couple to the electromagnetic mode, resulting in an increased collective coupling strength of the molecular excitons with the SLRs.

\section{STRONG COUPLING OF EXCITONS TO SLRS}

One of the signatures of strong light-matter coupling is the modification of the energy dispersion and the appearance of an anticrossing at the energy and momentum where the dispersions of the lattice modes and molecular excitonic transition cross each other. To measure the strong coupling between the molecular excitons and the SLRs, we have implemented angle-resolved measurements in order to measure the optical extinction as a function of the angle of incidence, i.e., as a function of the wave vector parallel to the surface of the array. These measurements retrieve the dispersive behavior of the resonances supported by the array. The collective nature of the strong coupling implies that the strength of the lightmatter interaction should increase by increasing the number of the excitons within the mode volume of the cavity, leading to an enhanced anticrossing in the dispersion. The coupling strength in the collective strong coupling is given by the Rabi energy:

$$
\hbar \Omega=2 \vec{E} \cdot \vec{d} \sqrt{N}
$$

where $\vec{E}$ and $\vec{d}$ are the electromagnetic field amplitude and the transition dipole moment of the exciton, respectively, and $N$ is the number of excitons coupled to the optical mode that expresses the collective nature of strong coupling. ${ }^{29}$

To strongly couple SLRs to molecular excitons, we spincoated a layer of PMMA doped with the dye molecules at 35 and $50 \mathrm{wt} \%$. In these experiments we select the periodicity of the lattice such that the energy of the SLRs at zero momentum, 

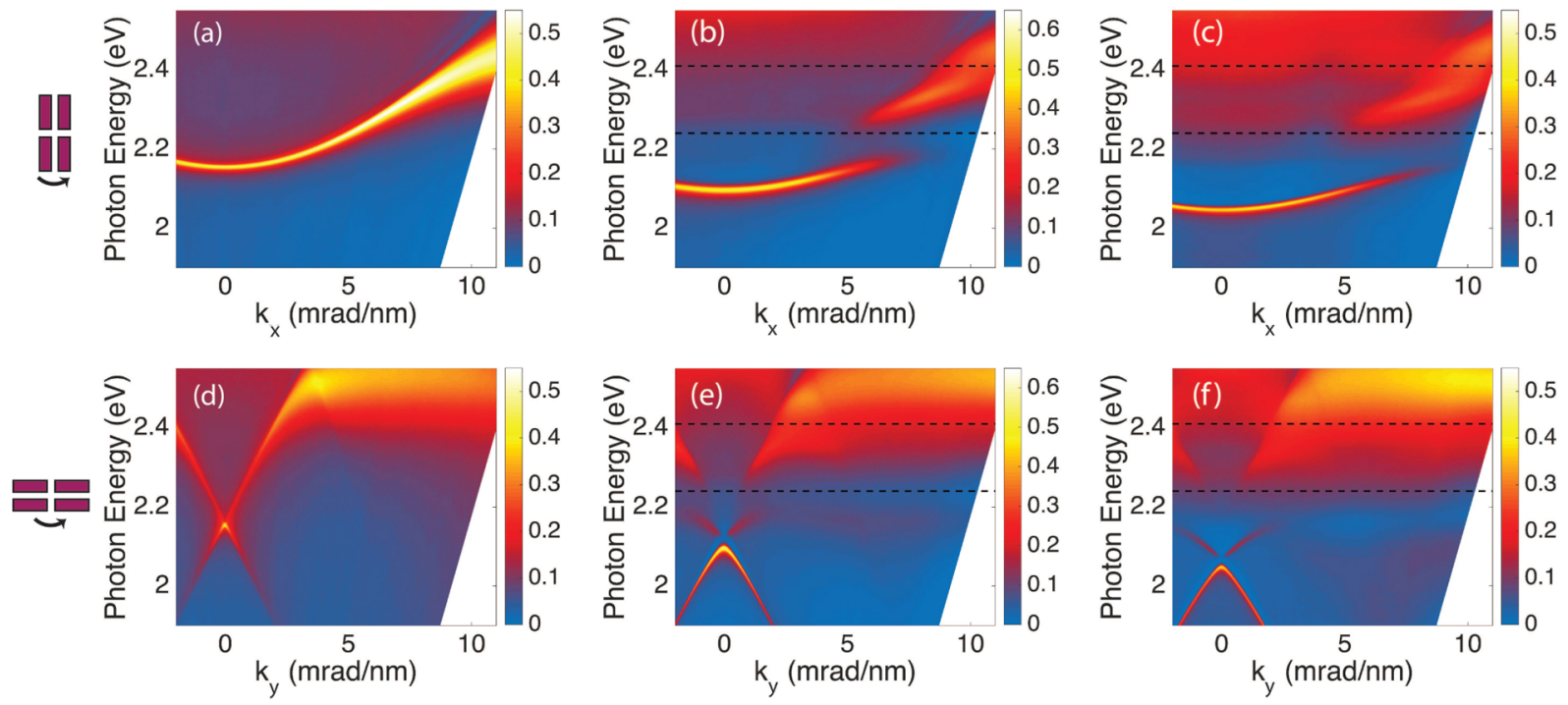

Figure 3. Measurements of the extinction for the array of nanoparticles with SLR excited along the $(0, \pm 1)$ RAs while the array is covered with 120 $\mathrm{nm}(\mathrm{a}, \mathrm{d})$ of undoped PMMA and doped PMMA with (b, e) $35 \mathrm{wt} \%$ and $(\mathrm{c}, \mathrm{f}) 50 \mathrm{wt} \%$ dye. (a-c) SLR and PEP dispersions when excited along the $x$-direction. $(\mathrm{d}-\mathrm{f})$ SLR and PEP dispersions along the $y$-direction. Black dashed lines indicate the excitonic and vibronic transition of the dye.

i.e., $k=0$, is lower than the energy of the molecular excitonic transition $\left(E_{X_{1}}=2.240 \mathrm{eV}\right)$. This condition corresponds to negative detuning between the energies of the cavity mode and the excitonic transition. The directions of the sample rotation to determine the SLRs from the extinction measurements are shown in the left side of Figure 3. In these measurements, the polarization of the incident light is fixed along the short axis of the nanoparticles, i.e., the $x$-axis. The extinction measurements are shown in Figure 3, where (a), (b), and (c) correspond to the sample rotation along the long nanoparticle axis, i.e., the $y$ axis, and (d), (e), and (f) along the $x$-axis. Figure $3(\mathrm{a})$ and (d) correspond to the extinction of a sample with a polymer layer of $120 \mathrm{~nm}$ but without the organic molecules. With these measurements we resolve the bare SLRs resulting from the different orders of diffraction. The grating equation is used as follows to correlate the extinction measurements with SLRs resulting from different RAs,

$$
\pm \vec{k}_{\| \mathrm{d}}=\vec{k}_{\| \mathrm{i}}+\vec{G}
$$

where $\vec{k}_{\| \mathrm{i}}=\frac{2 \pi}{\lambda} \sin (\theta) \hat{u}_{\mathrm{i}}$ and $\vec{k}_{\| \mathrm{d}}=\frac{2 \pi}{\lambda} n_{\text {eff }} \hat{u}_{\mathrm{d}}$ are the parallel components of the incident and diffracted wave vectors, respectively $\left(\hat{u}_{\mathrm{d}}=\left(\hat{u}_{x}, \hat{u}_{y}\right)\right.$ and $\hat{u}_{\mathrm{i}}$ are the unitary vectors along the diffracted and incident directions), $\theta$ is the angle between the wave vector of the incident beam and the direction normal to the surface of the sample, $n_{\text {eff }}$ is the effective index defining the phase velocity of the in-plane diffracted wave, i.e., the RAs, and $\vec{G}=\left[\left(2 \pi / a_{x}\right) p,\left(2 \pi / a_{y}\right) q\right]$ is the reciprocal lattice vector of the array with $p$ and $q$ corresponding to the orders of diffraction and $a_{x}$ and $a_{y}$ the lattice constants. In Figure 3(a), we observe the degenerate SLRs along $p=0, q= \pm 1$, i.e., the $(0, \pm 1)$ order. The dispersion of the $(0, \pm 1)$ RAs is given by

$$
E_{(0, \pm 1)}= \pm \frac{\hbar c}{n_{\mathrm{eff}}} \sqrt{k_{x}{ }^{2}+\left(2 \pi / a_{y}\right)^{2}}
$$

where $a_{y}=380 \mathrm{~nm}$ and $\vec{k}_{\| \mathrm{i}}=k_{x} \hat{u}_{x}$ has only a component along the $x$-direction; that is, the sample is rotated along the $y$ direction. The measured full width at half-maximum (fwhm) of this SLR is $16 \mathrm{meV}$ at $k_{x}=0 \mathrm{mrad} / \mathrm{nm}$. The fwhm of the SLR increases by increasing $k_{x}$ due to the reduction of the detuning between the RAs and the LSPR (at $E=2.458 \mathrm{eV}$ ), which results in an enhanced plasmonic character of the SLR. Moreover, one should notice that the dispersion of the SLR along $k_{x}$ and $k_{y}$ is quite different, a difference that also extends to the line widths. For this particular system, it seems that the SLR along $k_{x}$ is more damped than along $k_{y}$. As the resonances in these systems have Fano lineshapes, one can also describe the features of the resonance such as line width by the strength of the coupling between the discrete state (RAs) and the continuum (LSPRs), which depends on the dispersion of the RAs.

Figure 3(b) and (c) correspond to the extinction of the samples with 35 and 50 wt \% dye concentration, respectively. In these figures, the energies of the excitonic and vibronic transitions of the molecules are marked by black dashed lines at $E_{X_{1}}=2.24 \mathrm{eV}$ and $E_{X_{2}}=2.41 \mathrm{eV}$, respectively. A clear anticrossing between the excitonic resonance and the SLR can be observed. This anticrossing gets more pronounced as the molecular concentration is increased. In addition, the onset of hybridization between vibronic molecular transitions and the SLR, which leads to the formation of the middle polariton, is visible at $E \approx 2.41 \mathrm{eV}$, although the coupling strength is not large enough to give rise to a clear splitting.

The other RAs that provide a new set of SLRs with entirely different dispersion compared to the previous case are the RAs arising from the diffraction of $\vec{k}_{\| \mathrm{i}}$ along the $y$-direction, i.e., $\vec{k}_{\| \mathrm{i}}=$ $k_{y} \hat{u}_{y}$. This situation corresponds in the experiments to rotating the sample along the $x$-direction. In this case, the dispersion of the $(0, \pm 1)$ diffraction orders are linear and given by

$$
E_{(0, \pm 1)}=\mp \frac{\hbar c}{n_{\text {eff }}}\left[k_{y} \pm 2 \pi / a_{y}\right]
$$

The SLRs with linear dispersions are displayed in Figure 3(d). Similar to the previous case, the strong coupling of the SLRs with the molecular excitons, shown in Figure 3(e) and (f) for 35 and 50 wt \% dye concentration, respectively, leads to their hybridization and the formation of PEPs with an anticrossing at $k_{y} \approx 1.5 \mathrm{mrad} / \mathrm{nm}$. As we show ahead, one important result of this simultaneous occurrence of PEPs with 

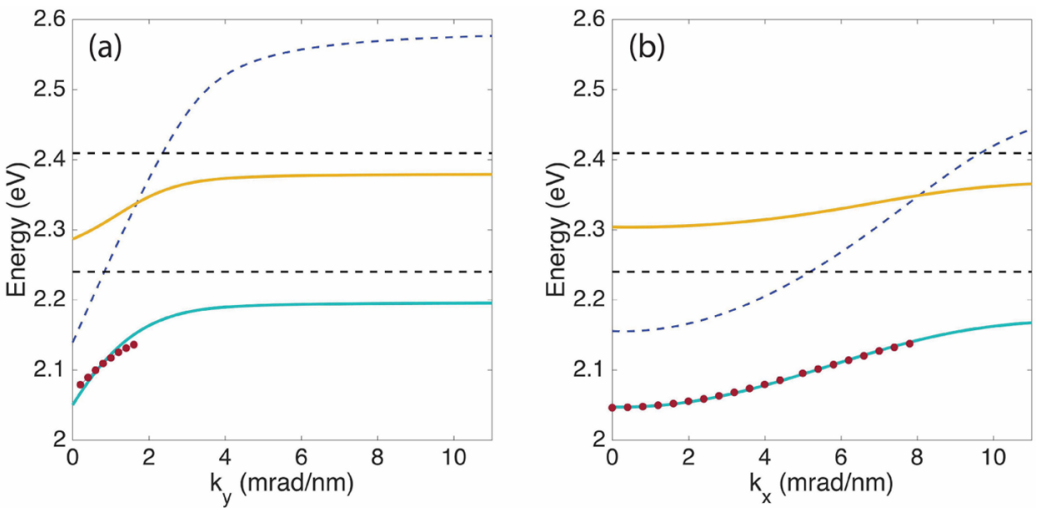

Figure 4. Dispersions of the lower polaritons (red dots) obtained from the extinction maps for SLRs propagating along the (a) $y$ - and (b) $x$ direction. The solid green and yellow lines represent the polaritons resulting from the analytical model based on the described Hamiltonian. The black dashed line at $E_{X_{1}}=2.24 \mathrm{eV}\left(E_{X_{2}}=2.41 \mathrm{eV}\right)$ represents the energy of the excitonic (vibronic) transition in the molecules. The blue dashed line represents the energy dispersion of SLRs in the absence of dye molecules.

(a)

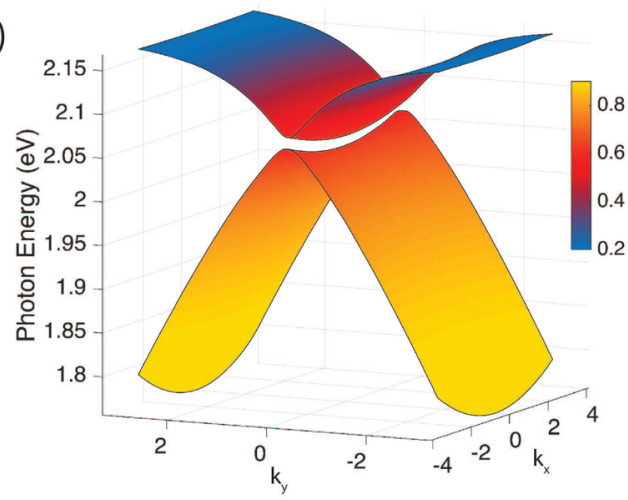

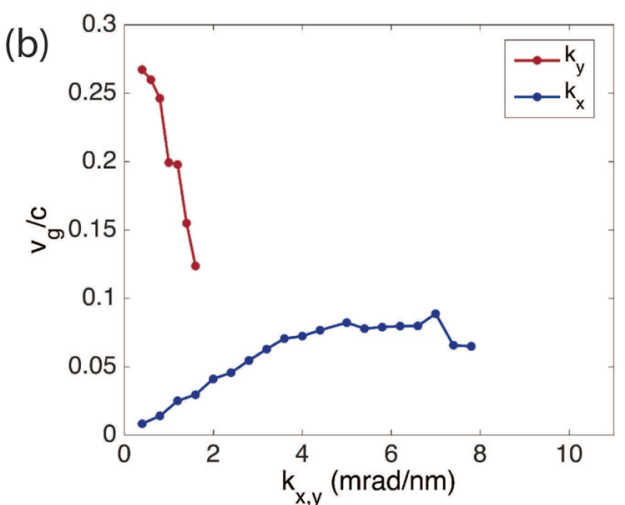

Figure 5. (a) Three-dimensional dispersion of PEPs. The color indicates the photon fraction of the PEPs. The photon fraction of $\sim 0.5$ around the Rabi splitting indicates the strong plasmonic behavior of the exciton-polaritons. (b) Group velocity of PEPs as a function of $k_{x}$ (blue circles) and $k_{y}$ (red circles).

distinct dispersion anisotropy is the modified PEP group velocity. An interesting feature that can be seen in the dispersion of the strongly coupled linear SLRs to excitons is the dark nature (absence of extinction) of the upper polariton band at $k_{y}=0 \mathrm{mrad} / \mathrm{nm}$. A similar response has been reported previously, and it is explained by the antisymmetric field and charge distributions across the nanoparticles due to the optical retardation along their long axis. ${ }^{30,31}$ These distributions lead to the suppression of the net dipole moment and the absence of extinction. Recent experiments showing exciton-polariton lasing in nanoparticle arrays have revealed the relevance of dark modes for PEP condensation. ${ }^{4}$ We note also that the SLRs associated with the $( \pm 1,0)$ RAs are not visible in the extinction measurements of Figure 3. This absence is due to the shorter lattice constant along the $x$-direction $\left(a_{x}=200 \mathrm{~nm}\right)$, which shifts these resonances to higher frequencies.

To determine the coupling strength and group velocities of PEPs along different directions, we can fit the measurements to a few-level Hamiltonian given by

$$
H=\left(\begin{array}{ccc}
E_{\mathrm{SLR}} & \hbar \Omega_{1} & \hbar \Omega_{2} \\
\hbar \Omega_{1} & E_{X 1} & 0 \\
\hbar \Omega_{2} & 0 & E_{X 2}
\end{array}\right)
$$

where $E_{\text {SLR }}$ is the energy of the SLR and $E_{X 1}$ and $E_{X 2}$ are the energies of the vibronic bands. Here, we focus on the linear- response regime, such that the two dominant vibronic peaks can be treated as separate independent resonances of the molecule. Moreover, $\hbar \Omega_{1}$ and $\hbar \Omega_{2}$ are the Rabi energies describing the coupling between the SLR and the molecular transitions. Note that in this model we treat the different SLRs independently from each other. By diagonalizing this Hamiltonian, we can obtain the energy eigenvalues of the system. In Figure 4(a,b), the green solid lines correspond to the fitted dispersions to the measured lower PEP bands for the sample with 50 wt \% dye concentration. The red dots in these figures represent the experimental values obtained from the measurements of Figure 3(c,f). A good agreement between the measured and calculated dispersions is achieved. However, there are some discrepancies that can be related to the simplified nature of the model and the fact that it does not take into account all the molecular energy sublevels and transitions that can also couple to the SLRs. In addition, in the energy region where the upper polariton modes are expected, there are a number of additional photonic modes in the system (e.g., guided modes in the polymer layer). As we have not included these modes in the simple model we use to obtain the lower polariton dispersion, the middle and upper polaritons of the model Hamiltonian do not correspond well to the actual modes of the system. Therefore, we do not discuss their dispersions in detail. 
Using these fits we extract the value of $\sim 120 \mathrm{meV}$ for the Rabi energy of the $(0, \pm 1)$ PEPs with 35 wt \% dye concentration, which is greater than the line widths of SLR $(77 \mathrm{meV})$ and the inhomogeneously broadened excitonic transition $(90 \mathrm{meV})$. By increasing the dye concentration to 50 wt \% we observe that the Rabi splitting increases to $\sim 170 \mathrm{meV}$ for PEPs along $k_{x}$ and $k_{y}$.

To emphasize the anisotropy on the dispersion of the PEPs, we have reconstructed the three-dimensional PEP dispersion in Figure 5(a). The dispersions are extracted from the diagonalization of the few-level Hamiltonian given by eq 7 . The photon fraction of the PEPs is color-coded in Figure 5(a). A close look into this figure shows that the curvature of the dispersion surfaces is different along different directions. In comparison with microcavities that manifest cylindrical symmetry and isotropic dispersion, arrays of nanoparticles can have very anisotropic dispersion. One immediate consequence of this anisotropic dispersion is the different group velocity $\left(v_{\mathrm{g}}=\hbar^{-1}(\mathrm{~d} E / \mathrm{d} k)\right)$ of the polaritons along different directions. This feature can be particularly interesting within the context of enhanced exciton-polariton transport in the strong coupling limit as a way to control the flow of exciton-polaritons. ${ }^{5,6}$ The anisotropic nature of the PEPs will also enable controlling the scattering of the exciton-polaritons applicable for exciton-polariton condensation and parametric oscillation and amplification. ${ }^{4}$

In order to determine the PEPs' group velocities along the $x$ and $y$ directions, we have calculated the first derivate of the dispersion from the experimental extinction maxima. The group velocities of the PEPs, normalized by the speed to light in a vacuum, are shown in Figure 5(b) as a function of the wave vector. In this figure, the red curve corresponds to the group velocity of the lower PEP band along $k_{y}$, while the blue curve shows the group velocity along $k_{x}$. The anisotropy in the dispersion leads to very different group velocities, with a velocity ratio close to 10 for small values of the wave vector. While the close-to-linear dispersion for $k_{y}$ leads to a large group velocity, the parabolic dispersion for $k_{x}$ leads to slow PEPs. In addition to the different dispersions along different directions, the difference in the curvature of the PEPs should lead to distinct effective masses and anisotropic transport of excitonpolaritons. For SLRs arising from the diffraction along $x$ direction, the effective mass can be calculated from the second derivative of the dispersion $\left(m^{*}=4 \times 10^{-4} m_{\mathrm{e}}\right.$ at $k_{x}=0 \mathrm{mrad} /$ $\mathrm{nm}$, where $m_{\mathrm{e}}$ is the electron mass). However, for SLRs along the $y$-direction, the dispersions are linear and the effective mass of the relativistic particles needs to be taken into account.

\section{CONCLUSION}

In conclusion, we have shown that planar arrays of metallic nanoparticles can provide a powerful platform for creating the hybrid states of light and matter, plasmon-exciton-polaritons, with distinct dispersions and enable making a polaritonic medium with tailored anisotropy. The quality of the fabricated arrays and their associated geometrical parameters lead to the observation of Rabi splitting between the upper and lower PEPs with different polariton dispersions and group velocities. Such degree of control of the properties of PEPs can be potentially applicable for the design of polariton-based circuits with an anisotropic response.

\section{AUTHOR INFORMATION}

\section{Corresponding Author}

*E-mail: m.ramezani@differ.nl.

ORCID

Mohammad Ramezani: 0000-0003-2781-0217

Johannes Feist: 0000-0002-7972-0646

Antonio I. Fernández-Domínguez: 0000-0002-8082-395X

Notes

The authors declare no competing financial interest.

\section{ACKNOWLEDGMENTS}

This research was financially supported by the Nederlandse Organisatie voor Wetenschappelijk Onderzoek (NWO) through the project LEDMAP of the Technology Foundation STW and through the Industrial Partnership Program Nanophotonics for Solid State Lighting with Philips. This work has also been funded by the European Research Council under grant agreements ERC-2011-AdG-290981 and ERC-2016STG-714870 and by the Spanish MINECO under contracts MAT2014-53432-C5-5-R, FIS2015-64951-R, and the "Maria de Maeztu" program for Units of Excellence in R\&D (MDM2014-0377). We thank Marc A. Verschuuren for fabrication of the sample. We also thank S. R. K. Rodriguez for the discussions.

\section{REFERENCES}

(1) Kéna-Cohen, S.; Forrest, S. R. Room-temperature polariton lasing in an organic single-crystal microcavity. Nat. Photonics 2010, 4, 371-375.

(2) Plumhof, J. D.; Stöferle, T.; Mai, L.; Scherf, U.; Mahrt, R. F. Room-temperature Bose-Einstein condensation of cavity excitonpolaritons in a polymer. Nat. Mater. 2014, 13, 247-252.

(3) Daskalakis, K. S.; Maier, S. A.; Murray, R.; Kéna-Cohen, S. Nonlinear interactions in an organic polariton condensate. Nat. Mater. 2014, 13, 271-278.

(4) Ramezani, M.; Halpin, A.; Fernández-Domínguez, A. I.; Feist, J.; Rodriguez, S. R.-K.; Garcia-Vidal, F. J.; Rivas, J. G. Plasmon-excitonpolariton lasing. Optica 2017, 4, 31-37.

(5) Feist, J.; Garcia-Vidal, F. J. Extraordinary Exciton Conductance Induced by Strong Coupling. Phys. Rev. Lett. 2015, 114, 196402.

(6) Schachenmayer, J.; Genes, C.; Tignone, E.; Pupillo, G. CavityEnhanced Transport of Excitons. Phys. Rev. Lett. 2015, 114, 196403.

(7) Orgiu, E.; George, J.; Hutchison, J. A.; Devaux, E.; Dayen, J. F.; Doudin, B.; Stellacci, F.; Genet, C.; Schachenmayer, J.; Genes, C.; Pupillo, G.; Samori, P.; Ebbesen, T. W. Conductivity in organic semiconductors hybridized with the vacuum field. Nat. Mater. 2015, 14, 1123-1129.

(8) Spano, F. C. Optical microcavities enhance the exciton coherence length and eliminate vibronic coupling in J-aggregates. J. Chem. Phys. 2015, 142, 184707.

(9) Galego, J.; Garcia-Vidal, F. J.; Feist, J. Cavity-Induced Modifications of Molecular Structure in the Strong-Coupling Regime. Phys. Rev. X 2015, 5, 041022.

(10) Hutchison, J. A.; Schwartz, T.; Genet, C.; Devaux, E.; Ebbesen, T. W. Modifying Chemical Landscapes by Coupling to Vacuum Fields. Angew. Chem., Int. Ed. 2012, 51, 1592-1596.

(11) Kowalewski, M.; Bennett, K.; Mukamel, S. Cavity Femtochemistry: Manipulating Nonadiabatic Dynamics at Avoided Crossings. J. Phys. Chem. Lett. 2016, 7, 2050-2054.

(12) Deng, H.; Haug, H.; Yamamoto, Y. Exciton-polariton BoseEinstein condensation. Rev. Mod. Phys. 2010, 82, 1489-1537.

(13) Zengin, G.; Wersäll, M.; Nilsson, S.; Antosiewicz, T. J.; Käll, M.; Shegai, T. Realizing Strong Light-Matter Interactions between SingleNanoparticle Plasmons and Molecular Excitons at Ambient Conditions. Phys. Rev. Lett. 2015, 114, 157401-6. 
(14) Bellessa, J.; Bonnand, C.; Plenet, J. C.; Mugnier, J. Strong Coupling between Surface Plasmons and Excitons in an Organic Semiconductor. Phys. Rev. Lett. 2004, 93, 036404-4.

(15) Memmi, H.; Benson, O.; Sadofev, S.; Kalusniak, S. Strong Coupling between Surface Plasmon Polaritons and Molecular Vibrations. Phys. Rev. Lett. 2017, 118, 126802.

(16) Dintinger, J.; Klein, S.; Bustos, F.; Barnes, W. L.; Ebbesen, T. W. Strong coupling between surface plasmon-polaritons and organic molecules in subwavelength hole arrays. Phys. Rev. B: Condens. Matter Mater. Phys. 2005, 71, 035424.

(17) Rodriguez, S.; Rivas, J. G. Surface lattice resonances strongly coupled to Rhodamine 6G excitons: tuning the plasmon-excitonpolariton mass and composition. Opt. Express 2013, 21, 27411-27421.

(18) Väkeväinen, A. I.; Moerland, R. J.; Rekola, H. T.; Eskelinen, A. P.; Martikainen, J. P.; Kim, D. H.; Törmä, P. Plasmonic Surface Lattice Resonances at the Strong Coupling Regime. Nano Lett. 2014, 14, $1721-1722$.

(19) Guo, K.; Lozano, G.; Verschuuren, M. A.; Rivas, J. G. Control of the external photoluminescent quantum yield of emitters coupled to nanoantenna phased arrays. J. Appl. Phys. 2015, 118, 073103.

(20) Chikkaraddy, R.; de Nijs, B.; Benz, F.; Barrow, S. J.; Scherman, O. A.; Rosta, E.; Demetriadou, A.; Fox, P.; Hess, O.; Baumberg, J. J. Single-molecule strong coupling at room temperature in plasmonic nanocavities. Nature 2016, 535, 127-130.

(21) Rodriguez, S. R. K.; Feist, J.; Verschuuren, M. A.; Garcia Vidal, F. J.; Gómez Rivas, J. Thermalization and Cooling of Plasmon-Exciton Polaritons: Towards Quantum Condensation. Phys. Rev. Lett. 2013, 111, 166802.

(22) Shi, L.; Hakala, T. K.; Rekola, H. T.; Martikainen, J.-P.; Moerland, R. J.; Törmä, P. Spatial Coherence Properties of Organic Molecules Coupled to Plasmonic Surface Lattice Resonances in the Weak and Strong Coupling Regimes. Phys. Rev. Lett. 2014, 112, 153002 .

(23) Eizner, E.; Avayu, O.; Ditcovski, R.; Ellenbogen, T. Aluminum Nanoantenna Complexes for Strong Coupling between Excitons and Localized Surface Plasmons. Nano Lett. 2015, 15, 6215-6221.

(24) Humphrey, A. D.; Barnes, W. L. Plasmonic surface lattice resonances on arrays of different lattice symmetry. Phys. Rev. B: Condens. Matter Mater. Phys. 2014, 90, 075404.

(25) Ramezani, M.; Lozano, G.; Verschuuren, M. A.; Gómez-Rivas, J. Modified emission of extended light emitting layers by selective coupling to collective lattice resonances. Phys. Rev. B: Condens. Matter Mater. Phys. 2016, 94, 125406.

(26) Verschuuren, M. A. Substrate Conformal Imprint Lithography for Nanophotonics. Ph.D. thesis, Utrecht University, 2010.

(27) Auguié, B.; Barnes, W. L. Collective Resonances in Gold Nanoparticle Arrays. Phys. Rev. Lett. 2008, 101, 143902.

(28) Vecchi, G.; Giannini, V.; Gómez Rivas, J. Shaping the Fluorescent Emission by Lattice Resonances in Plasmonic Crystals of Nanoantennas. Phys. Rev. Lett. 2009, 102, 146807.

(29) Wang, S.; Chervy, T.; George, J.; Hutchison, J. A.; Genet, C.; Ebbesen, T. W. Quantum Yield of Polariton Emission from Hybrid Light-Matter States. J. Phys. Chem. Lett. 2014, 5, 1433-1439.

(30) Rodriguez, S. R. K.; Abass, A.; Maes, B.; Janssen, O. T. A.; Vecchi, G.; Gómez Rivas, J. Coupling Bright and Dark Plasmonic Lattice Resonances. Phys. Rev. X 2011, 1, 021019.

(31) Abass, A.; Rodriguez, S. R.-K.; Rivas, J. G.; Maes, B. Tailoring Dispersion and Eigenfield Profiles of Plasmonic Surface Lattice Resonances. ACS Photonics 2014, 1, 61-68. 